\title{
Significance of MRI/Transrectal Ultrasound Fusion Three-Dimensional Model-Guided, Targeted Biopsy Based on Transrectal Ultrasound-Guided Systematic Biopsy in Prostate Cancer Detection: A Systematic Review and Meta-Analysis
}

\author{
Yongquan Tang Zhihong Liu Liangyou Tang Ruochen Zhang Yiping Lu \\ Jiayu Liang Zijun Zou Chuan Zhou Yujie Wang \\ Department of Urology/Institute of Urology, West China School of Medicine/West China Hospital, Sichuan University, \\ Chengdu, China
}

\section{Keywords}

Magnetic resonance imaging · Prostate biopsy - Prostate cancer $\cdot$ Transrectal ultrasound

\footnotetext{
Abstract

Purpose: To assess MRI/Transrectal Ultrasound (TRUS) fusion three-dimensional model-guided targeted biopsy (3D-Tb) versus TRUS-guided systematic biopsy (Sb) in detecting overall and high-Gleason-score $(\geq 7)$ prostate cancer (PCa). Methods: Pubmed and Web of science were searched. Studies with men having a suspicious lesion on MRI were included, which were divided into initial biopsy, previous negative biopsy, and mixed groups in meta-analysis. Results: Totally 13 cohorts in 12 studies, with 3,225 men were included. In total population, 3D-Tb and $\mathrm{Sb}$ did not differ significantly in the PCa detection rate ( 43.1 vs. $42.6 \%, p=0.36$ ), but after excluding initial biopsy group, the superiority of 3D-Tb became significant $(p=0.01) ; 3 \mathrm{D}-\mathrm{Tb}$ had a significantly higher detection rate of high-Gleason-score $\mathrm{PCa}$ compared to $\mathrm{Sb}$ (30.0 vs. $24.1 \%, p<0.05$ ); 3D-Tb plus Sb significantly improved the PCa detection rate based on Sb alone (52.7 vs. $42.6 \%, p<0.05)$. Conclusions: In men with increased serum
}

PSA and/or abnormal DRE and suspicious lesion on MRI but non-previous evidence of PCa, 3D-Tb plus Sb improves the PCa detection rate based on $\mathrm{Sb}$ alone. 3D-Tb alone has better performance in detecting high-Gleason-score PCa, and tends to have a higher PCa detection rate in population with previous negative biopsy compared to Sb.

(c) 2017 S. Karger AG, Basel

\section{Introduction}

The diagnosis of prostate cancer $(\mathrm{PCa})$ is principally based on transrectal ultrasound (TRUS)-guided systematic prostate biopsy ( $\mathrm{Sb})$, with around 12 cores in standard $[1,2]$. The criteria for men to undergo prostate biopsy include prostate-specific antigen (PSA) $>4 \mathrm{ng} / \mathrm{mL}$ and/or positive digital rectal examination (DRE) [3]. Because of low sensitivity of ultrasound to prostate tumor, ultrasound-guided prostate biopsies are generally mapping all around the prostate without targets [4]. However,

Y.T. and Z.L. contributed equally to the study.

\section{KARGER}

(c) 2017 S. Karger AG, Basel

E-Mail karger@karger.com

www.karger.com/uin 
a study showed that the sensitivity and specificity of TRUS-Sb were 47.3 and $92.5 \%$ respectively [5]. The efficacy did not improve even by increasing the number of cores $[6,7]$. Moreover, several studies found obvious discrepancy of the Gleason scores between prostate biopsy and prostatectomy specimens [8-10]. Compared to biopsy specimens, the Gleason score of prostatectomy specimens often upgrade in $21-53 \%$ of patients [11].

Multiparametric MRI consists of T2-weighted, diffusion-weighted and contrast-enhanced dynamic imaging. It is regarded as the most sensitive imaging modality in detecting prostate tumor [11]. So when combined with Multiparametric MRI, TRUS-guided biopsy may become more sensitive and targeted [12]. Recently, a novel device combined the previous MRI and real-time TRUS in computer to establish a three-dimension model of prostate and suspicious lesions within it. Guided by this model, both lesion-targeted biopsy (Tb) and systematic biopsy could be done [13]. So far, several studies have tested the diagnostic efficiency of MRI/TRUS fusion three-dimension (3D) model-guided targeted prostate biopsy (3D$\mathrm{Tb})$. However, compared to the traditional TRUS-guided 12-core prostate biopsy, many studies showed that the $3 \mathrm{D}-\mathrm{Tb}$ might have better sensitivity without statistical differences [14-18]. Furthermore, some researches showed that $3 \mathrm{D}-\mathrm{Tb}$ tended to find more high-Gleasonscore $(\geq 7)$ cases [16-18], but some other studies did not obtain significant results [14, 15, 19-22]. To our knowledge, rarely do studies test the efficiency of 3D-Tb combined with $\mathrm{Sb}$ as primary endpoint.

Three years ago, Schoots et al. [23] had made a similar meta-analysis. But their enrolled studies mostly performed targeted biopsy in MRI-TRUS 2D fusion model, which has a significantly different diagnostic capacity with 3D fusion [24]. Besides that meta-analysis had not assessed benefits of 3D-Tb combined with Sb. Since then, several studies with a large sample size have emerged. This study tries to integrate the data in previous studies to make a systematic review and meta-analysis to assess the advantages and limitations of the novel 3D-Tb compared with $\mathrm{Sb}$.

\section{Methods}

Objective

We primarily aimed to evaluate the relative diagnostic capacity of $3 \mathrm{D}-\mathrm{Tb}$ or $3 \mathrm{D}-\mathrm{Tb}$ plus $\mathrm{Sb}$ versus $\mathrm{Sb}$ in detecting the overall $\mathrm{PCa}$ and high/low-Gleason-score PCa. The comparison would be carried out in men for initial prostate biopsy and/or men with previous negative biopsy respectively.

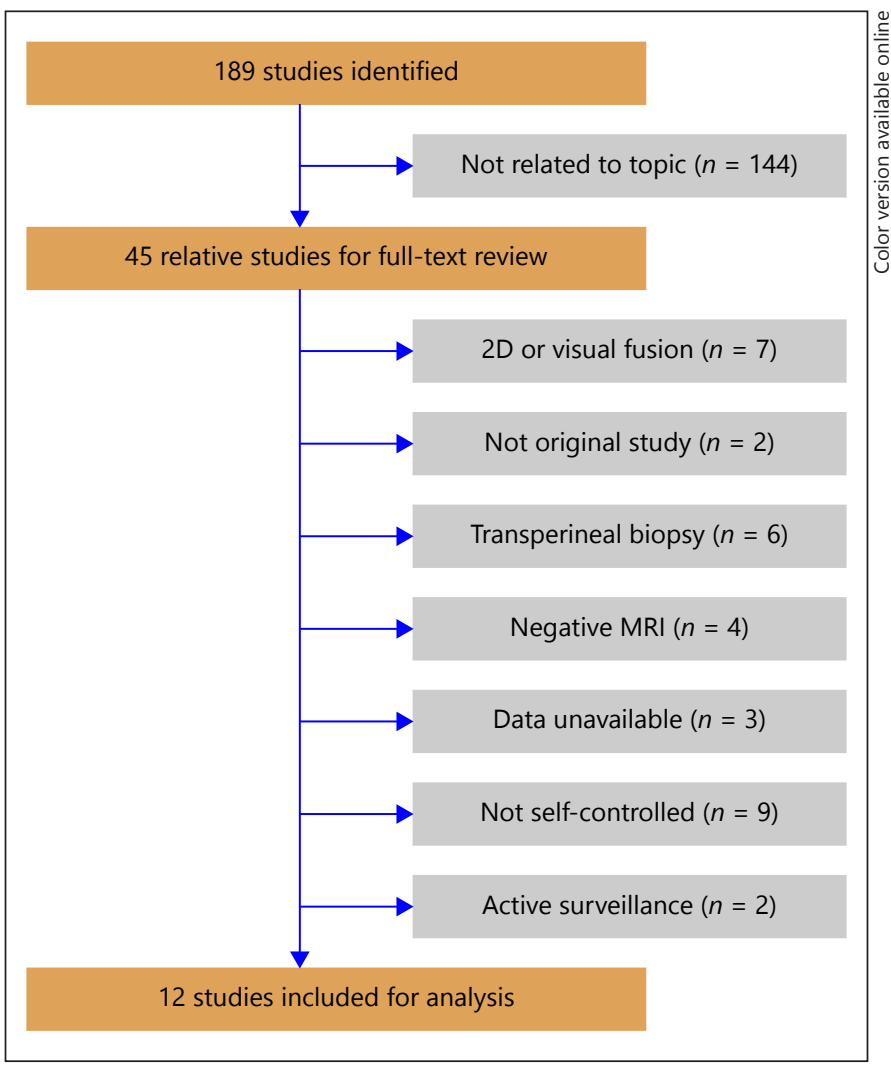

Fig. 1. Flow diagram for the selection process of the included studies.

Search strategy

The search terms were "prostate cancer," ("transrectal ultrasound" or "TRUS"), ("MRI") and ("fusion" or "targeted"). All the potential studies in the Pubmed and Web of Science would be screened, and the search was updated to February 3, 2017.

\section{Inclusion and Exclusion Criteria}

We included original studies, and men were scheduled to prostate biopsy for increased serum PSA (generally $\geq 4 \mathrm{ng} / \mathrm{L}$ ) and/or positive DRE, and had at least one suspicious lesion in prostate MRI prior to biopsy. In addition, the studies to be included must perform both $3 \mathrm{D}-\mathrm{Tb}$ and $\mathrm{Sb}$ transrectally in the same session, and intended to test the capacity of 3D-Tb in detecting PCa relative to traditional Sb (3D-Tb vs. Sb, and/or 3D$\mathrm{Tb}$ plus $\mathrm{Sb}$ vs. $\mathrm{Sb}$ ). The $\mathrm{Sb}$ must have standard 12 or very near 12 $(12 \pm 2)$ cores, and could be guided by TRUS or MRI/TRUS fusion 3D prostate model.

The study or population with any one of following criteria was excluded: (A) Unpublished data or without detail records; (B) Men with transperineal or other systematic protocol ( $\neq 12 \pm 2$ cores) biopsy; (C) Men with proven PCa on active surveillance; (D) Men without any suspicious lesion in previous MRI; (E) Tb was performed in MRI/TRUS 2D or visual fusion; (F) The 3D-Tb and Sb were not performed in the same men; $(G)$ Not primary study, including literatures review, meta-analysis and et al; $(\mathrm{H})$ Other conditions according to search results (Fig. 1). 
Studies Selection and Data Collection

The data collection was made by 2 reviewers independently. Whenever there were discrepancies, we re-reviewed and discussed. We first viewed the titles and abstracts for relevance to this topic. If not clear, we would turn to the full-text articles. The references cited by included studies were viewed as well for potentially relevant studies.

All eligible studies were imported into Endnote 7X. All full-text of included studies were reviewed. Data was extracted along with the publication information (author, year and journal), prospective/retrospective, population demography (country, mean age, mean serum PSA, mean prostate volume, number of positive DRE), MRI and biopsy strategy, initial/previous negative, outcomes, and other potential confounding data. When the overall population was not eligible, the subgroups that were relevant to the defined theme would be included. Numbers of the 4 principal items were extracted for meta-analysis: sample size, overall PCa (3D-Tb and/or $\mathrm{Sb}$ positive), respective $\mathrm{PCa}$ in $3 \mathrm{D}-\mathrm{Tb}$ and $\mathrm{Sb}$, and respective high/low-Gleason-score $\mathrm{PCa}$ in $3 \mathrm{D}-\mathrm{Tb}$ and $\mathrm{Sb}$. Considering the different definitions of clinical-significant or high-risk disease, such as Gleason score $\geq 4+3[11,17,22]$, Gleason score $\geq 7$ or Gleason score $=6$ and maximum cancer core length $>5 \mathrm{~mm}$ [15], or others $[20,21,25]$, we unified them as Gleason score $\geq 7[14,16$, 18 ] for more equitable analysis (Table 1$)$. On the other hand, tumors with Gleason pattern $4(3+4 / 4+3)$ have significantly worse prognosis than those with lower Gleason score [26, 27].

\section{Data Analysis}

Publication bias was visually assessed by inspecting the asymmetry of funnel plot, which was then tested using the Egger's test in Stata 14.0 (StataCorp LP, College Station, TX, USA). Studies would be included irrespective of whether the outcomes were significant or not. The heterogeneity would be tested by Review Manager 5.3 (Cochrane Community). When the $p$ value $>0.1$ and $I^{2}<50 \%$, the fixed-effect model would be employed, Otherwise, the random-effects model would be employed and then influence analysis would be conducted. The meta-analysis would be carried out by Review Manager 5.3. The method of Mantel-Haenszel in $95 \%$ CI, with OR was conducted as effects measure. When the $p$ value $>0.05$, the difference was regarded as statistically significant.

\section{Results}

\section{Description of Studies}

Totally 13 cohorts from 12 studies with 3,225 men (Table 1) were included for final analysis, of which Meng et al. [18] had 2 independent cohorts enrolling men for initial biopsy $(\mathrm{C} 1)$ and men with previous negative biopsy (C2) respectively. The included 13 cohorts were carried out in the United States, Canada, Japan, France, or Austria, and published from May 2012 to October 2016. Considering potential heterogeneity, those studies were divided into 3 sub-groups. Five cohorts only enrolled men for initial biopsy (group 1, 1,823 men), 3 cohorts only enrolled men with previous negative biopsy (group 3, 528 men), and 5 cohorts enrolled not only men for initial biopsy, but also men with previous negative biopsy (group 2, 874 men). In all studies, 3D-Tb and Sb were performed in the before-after self-controlled model. The main characteristics of included studies and men are presented in Table 1. Particularly, each man had at least 1 suspicious lesion on MRI. Each lesion was taken at least from one targeted core, but actually no less than 2 cores in most cases. In the same session, the Sb was mostly performed in the protocol of standard 12 cores.

\section{Publication Bias and Heterogeneity}

All comparisons did not show significant publication bias in funnel plots, which were confirmed by Egger's test $(p>0.05)$. The only data in comparison of $3 \mathrm{D}-\mathrm{Tb}$ versus $\mathrm{Sb}$ for overall PCa detection showed obvious heterogeneity $\left(p=0.03, I^{2}=48 \%\right)$, which was primarily influenced by the study of Ukimura et al. [19], then random effects were employed. The other comparisons $(p>0.05$ and $\left.I^{2} \leq 50 \%\right)$ employed the fixed effect model.

\section{D-Tb Combined with Sb versus Sb Alone in Overall $P C a$ Detection}

Totally 13 cohorts were included for this meta-analysis, with 3,225 men in all. 3D-Tb plus Sb detected 1,698 men $(52.7 \%)$ having PCa, significantly more than those detected by $\mathrm{Sb}$ alone $(1,375$ men, $42.6 \%$; $p<0.05)$. This result was in accordance with that of both subgroups. In cohorts enrolling men for initial biopsy, 3D-Tb plus $\mathrm{Sb}$ and $\mathrm{Sb}$ alone detected 1,023 and 877 men having PCa respectively ( 56.1 vs. $48.1 \%, p<0.05$ ). 3D-Tb combined with $\mathrm{Sb}$ and $\mathrm{Sb}$ alone detected 173 and $107 \mathrm{PCa}$, respectively, (32.8 vs. $20.3 \%, p<0.05)$ in cohorts with previous negative biopsy (Fig. 2).

\section{$3 D-T b$ versus Sb in Overall $P C a$ Detection}

The same 13 cohorts were included for this meta-analysis. 3D-Tb and Sb detected PCa in 1,389 men (43.1\%) and 1,375 men $(42.6 \%)$, respectively $(p=0.36)$, which did not show any statistic difference. In subgroups analyses, $3 \mathrm{D}-\mathrm{Tb}$ tended to detect less men with PCa in population for initial biopsy $(852 / 1,823$ vs. $877 / 1,823, p=0.08)$, but more men with $\mathrm{PCa}$ in population with previous negative biopsy $(133 / 528$ vs. $107 / 528, p=0.06)$. However, the differences were not statistically significant (Fig. 3).

\section{D-Tb versus Sb in High/Low-Gleason-Score PCa}

Detection

Totally 10 studies reporting on 2,573 men were included for this meta-analysis. 3D-Tb detected 771 men 


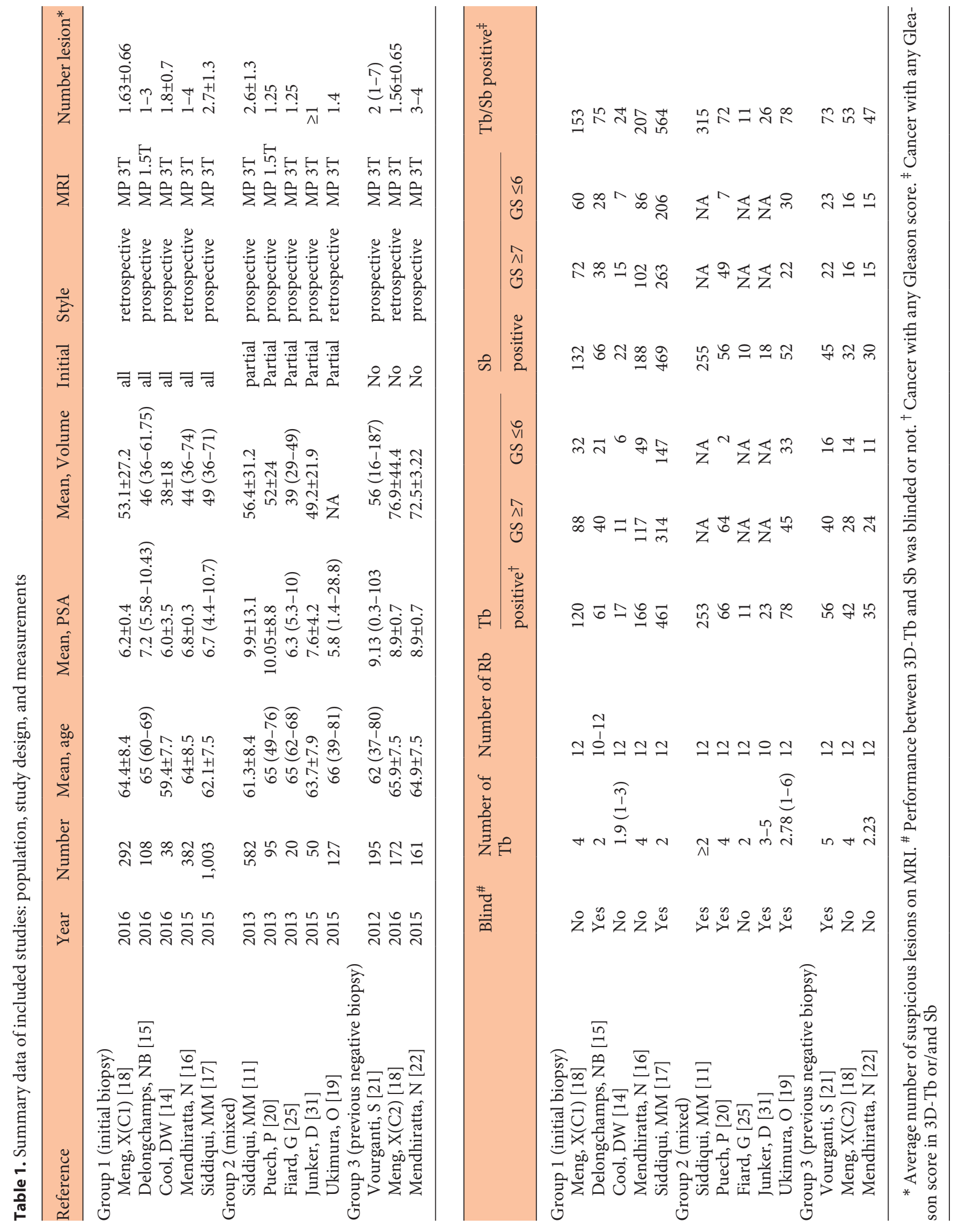




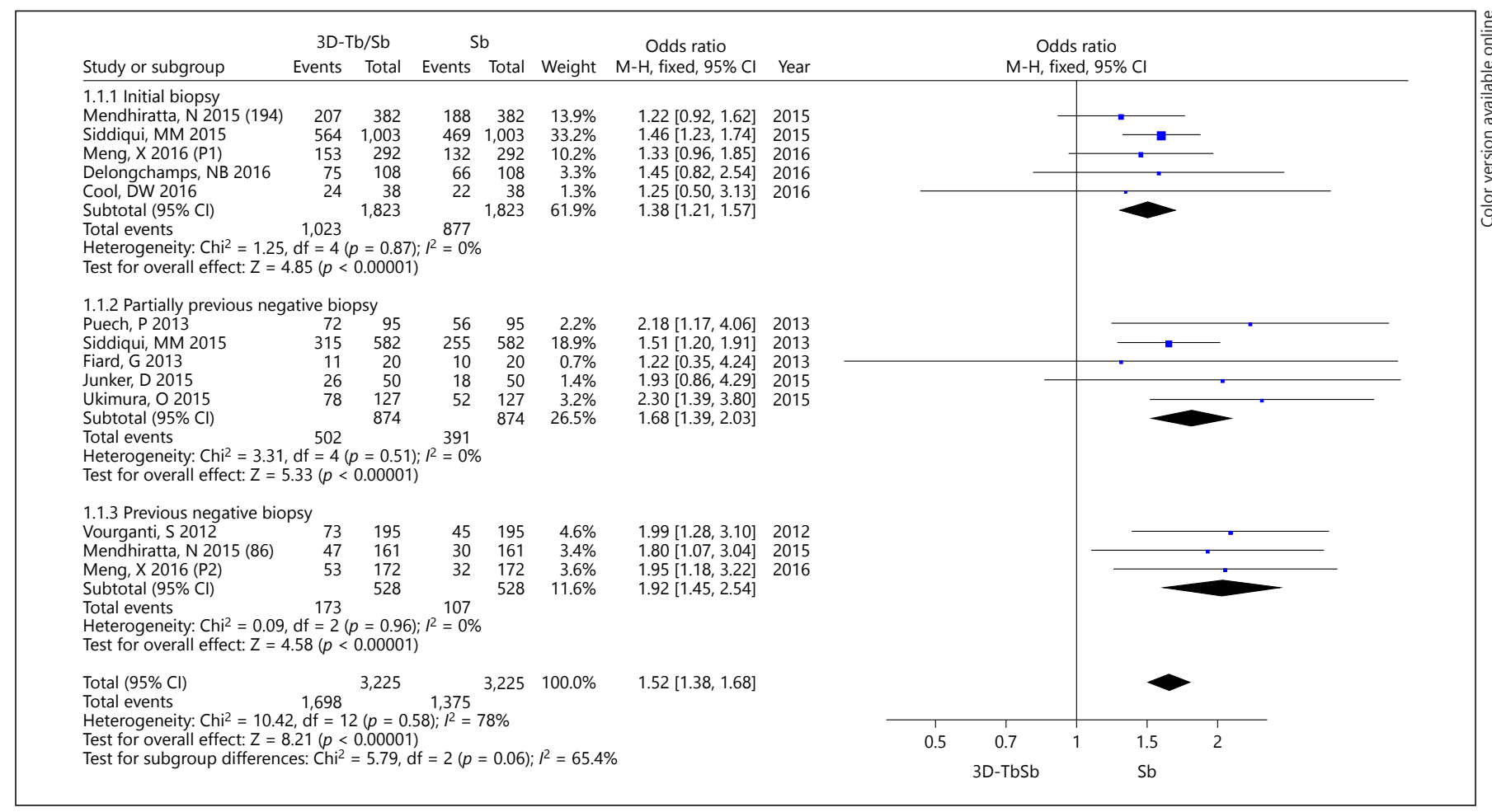

Fig. 2. Forest plot of overall prostate cancer diagnostic capacity of MRI/TRUS-fusion 3D model-guided targeted biopsy plus standard systematic biopsy $(\mathrm{Sb})$ versus $\mathrm{Sb}$ alone.

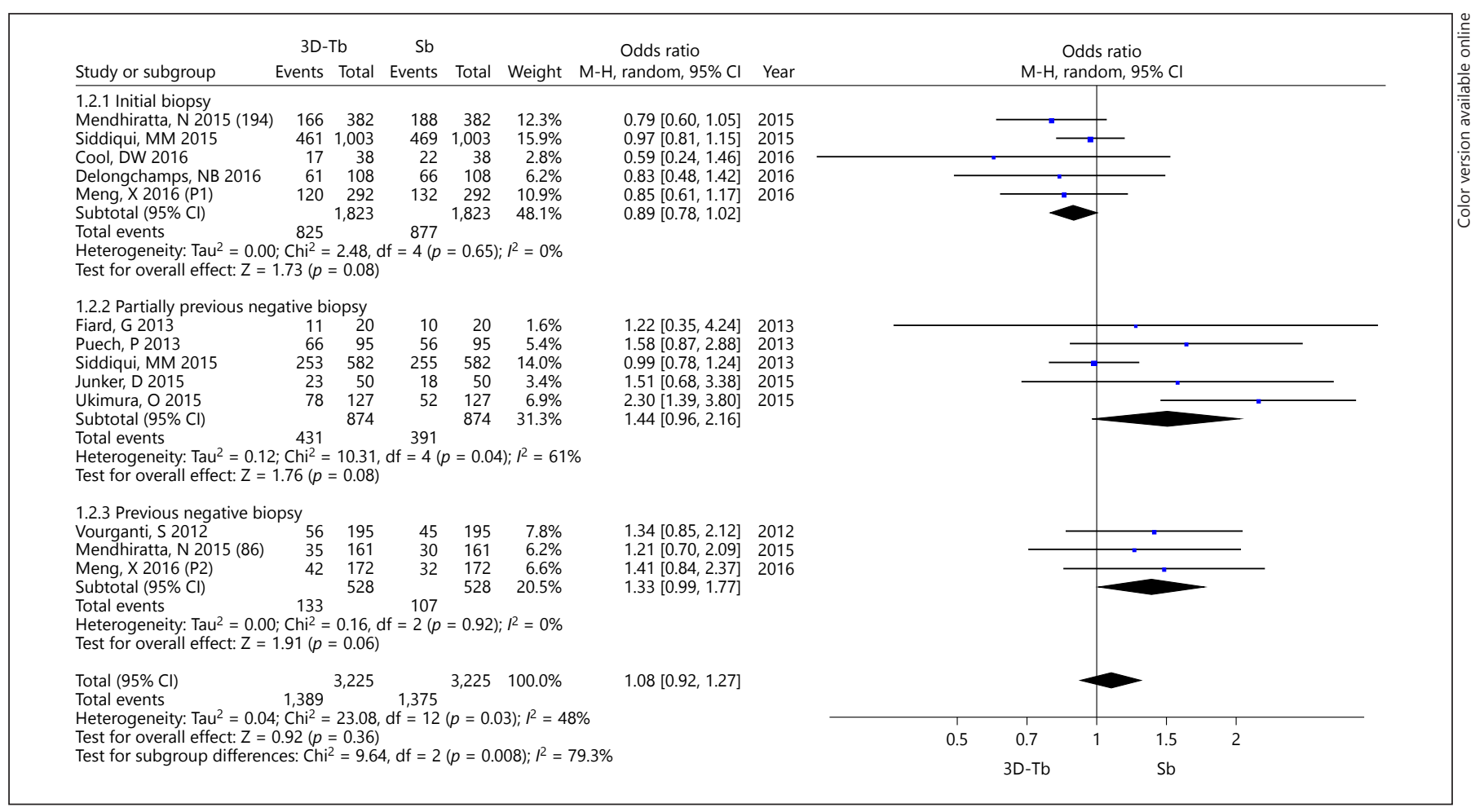

Fig. 3. Forest plot of overall prostate cancer diagnostic capacity of MRI/TRUS-fusion 3D model-guided targeted biopsy versus standard systematic biopsy. 
(30.0\%) with high-Gleason-score PCa, significantly more than those detected by Sb (621 men, 24.1\%; $p<$ $0.05)$. Very similar results were obtained in subgroups analyses. In population for initial biopsy, $3 \mathrm{D}-\mathrm{Tb}$ and $\mathrm{Sb}$ detected 570 men and 490 men with high-Gleasonscore PCa respectively ( 31.3 vs. $26.9 \%, p=0.004$ ). In the population with previous negative biopsy, $3 \mathrm{D}-\mathrm{Tb}$ and $\mathrm{Sb}$ detected 92 men and 53 men with high-Gleasonscore PCa respectively ( 17.4 vs. $10.0 \%, p=0.0006$; Fig. 4).

On the other hand, 3D-Tb detected 331 men (12.9\%) with low-Gleason-score PCa, significantly less than those detected by Sb (478 men, $18.58 \%, p<0.05)$. A very similar result was obtained in the population for initial biopsy. 3D-Tb and Sb detected 255 men (14.0\%) and 387 men $(21.2 \%)$ with low-Gleason-score PCa respectively $(p<0.05)$. However, in the population with previous negative biopsy, the difference was not significant, despite the fact that 3D-Tb detected less number of men with low-Gleason-score PCa (331 men vs. 478 men, $p=$ 0.16; Fig. 5.

\section{Discussion}

MRI/TRUS fusion 3D model not only takes advantages of the better sensitivity of MRI for detecting prostate tumor, but may also make the prostate biopsy targeted and easier to operate [28]. Of 2,137 men who were assigned to undergo MR-MRI in 4 studies, 1,794 men (84\%) returned with positive imaging finding $[14,16,17,19]$. This suggests that most PCa-suspected patients could go for MRI/TRUS fusion-targeted biopsy. However, as a novel technique, it may cost more financial resources. The golden standard in diagnosis of $\mathrm{PCa}$ is post-prostatectomy pathological grade. But just a proportion of patients are eligible to undergo prostatectomy, considering the elaborate surgical criteria including age, expected survival, PSA, pathological result of biopsy, and so on. As a result, most studies had to test the sensitivity and specificity relative to standard TRUS-guided 12-core Sb. In addition, most studies were before-after self-controlled, except that few studies were one-to-one matched [29] or grouping controlled $[24,30]$. Only the before-after, selfcontrolled study was included for analysis. The results and conclusions are just based on population scheduled to prostate biopsy for increased serum PSA and/or abnormal DRE, with suspicious lesion on MRI, but non-previous evidence of $\mathrm{PCa}$ (initial biopsy or previous negative biopsy).
Evaluation of Publication Bias and Heterogeneity

All eligible studies were included irrespective of language, state, journal, study size, outcomes and so on. We found no obvious publication bias by inspection of each funnel plots and Egger's test. In studies design, all included studies were same or very similar (Table 1), beforeafter self-controlled. So the selection bias may be negligible. The operation of $\mathrm{Sb}$ may be affected by knowledge of the results of 3D-Tb and vice versa (not blind), but the measurement was pathological grade, which was taken by pathologists. However, all studies did not reveal whether examination of specimens from $3 \mathrm{D}-\mathrm{Tb}$ and $\mathrm{Sb}$ was blinded to each other. In addition, approximately 10 studies were carried out in a single center. Therefore, it's lack of triple-blinded, multi-center, and randomized controlled trial with large population size. The Sb was guided by TRUS in 8 studies and by MRI/TRUS fusion 3D prostate model in 5 studies. To the latter, the core locations were produced automatically by the computer system, despite the suspicious lesions in the $3 \mathrm{D}$ prostate model, which aimed to mimic TRUS-guided biopsy.

\section{Evaluation of Outcomes}

Compared to traditional TRUS-guided Sb, MRI/TRUS fusion 3D-Tb did not show significantly different diagnostic capacity of $\mathrm{PCa}$ in the total population. However, 3D$\mathrm{Tb}$ detected more $\mathrm{PCa}$ in population with previous negative biopsy but fewer PCa in population for initial biopsy. These trends were not significant but agreed with the individual study in their own subgroups. Hence, it may need more studies or larger sample size to show the significant difference. Despite that, over $50 \%$ of patients in mixed cohorts had previous negative biopsy, when combined with cohorts for initial biopsy, the superiority of 3D-Tb became significant ( 40.2 vs. $35.5 \%, p=0.01)$. Therefore, $3 \mathrm{D}-\mathrm{Tb}$ probably has a higher $\mathrm{PCa}$ detection rate than that of $\mathrm{Sb}$ in population with previous negative biopsy.

When $3 \mathrm{D}-\mathrm{Tb}$ was combined with $\mathrm{Sb}$, the PCa detection rate significantly improved compared to $\mathrm{Sb}(p<0.05)$ or $3 \mathrm{D}-\mathrm{Tb}(p=0.000$, tested by Stata) alone, and similar results were obtained in populations of initial biopsy and previous negative biopsy. This trend was agreed with the most weighted study [17], and a prospective cohort study with a large sample size [30]. Therefore, just for the point of detection rate, $3 \mathrm{D}$ - $\mathrm{Tb}$ combined with $\mathrm{Sb}$ should be better than each of them alone in populations not only for initial biopsy and but also with previous negative biopsy.

Overall, in men with $\mathrm{PCa}, 3 \mathrm{D}-\mathrm{Tb}$ detected significantly more high-Gleason-score $(\geq 7) \mathrm{PCa}$ and significantly less low-Gleason-score $(\leq 6)$ PCa than those detected by TRUS-
62

Urol Int 2018;100:57-65 DOI: $10.1159 / 000484144$
Tang/Liu/Tang/Zhang/Lu/Liang/Zou/ Zhou/Wang 


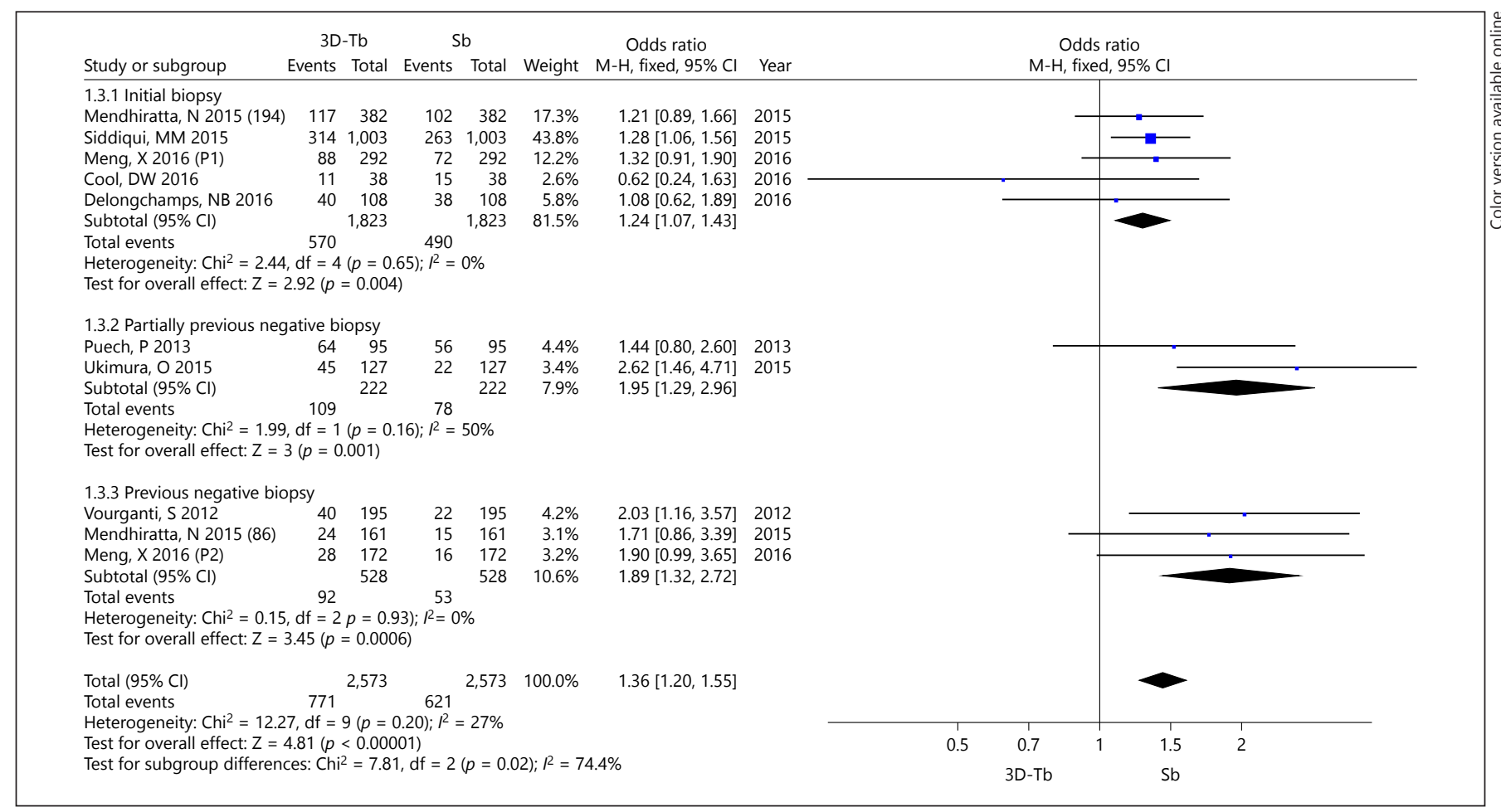

Fig. 4. Forest plot of high-Gleason-score prostate cancer diagnostic capacity of MRI/TRUS-fusion 3D model-guided targeted biopsy versus standard systematic biopsy.

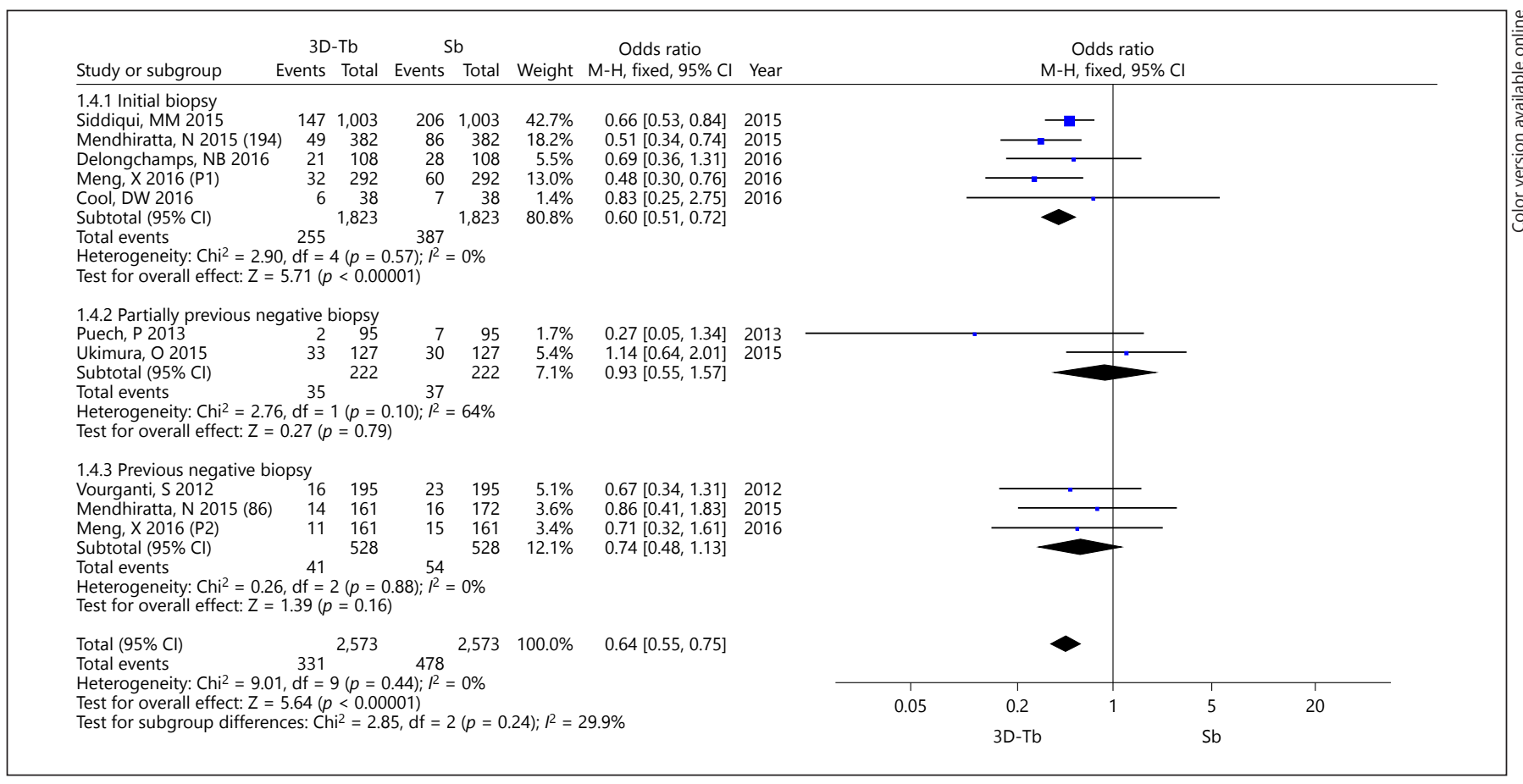

Fig. 5. Forest plot of low-Gleason-score prostate cancer diagnostic capacity of MRI/TRUS-fusion 3D model-guided targeted biopsy versus standard systematic biopsy. 
guided $\mathrm{Sb}$. In the population with previous negative biopsy, the inferiority of 3D-Tb in low-Gleason-score PCa detection was not significant, but it is reasonable considering that only 3 studies with 528 men were available for the subtotal analysis. We did not assess 3D-Tb combined with Sb versus 3D-Tb or Sb alone in the detection of high-Gleason-score PCa due to insufficient data. However, in a review of the literature, we found that both $3 \mathrm{D}-\mathrm{Tb}$ and $\mathrm{Sb}$ could miss high-Gleason-score PCa; it is probable that $3 \mathrm{D}-\mathrm{Tb}$ combined with $\mathrm{Sb}$ has better performance than each of them alone in detecting high-Gleason-score PCa. However, some researchers regarded $\mathrm{PCa}$ with Gleason score $\geq 8$ as high risk disease, but just 3 studies reporting on 430 patients provided exact data on this subject $[15,19,21]$. Among these patients, 3D-Tb and $\mathrm{Sb}$ found 27 and $29 \mathrm{PCa}$ with Gleason score $\geq 8$ respectively $(p=0.78$ ). This result is doubtful considering its small sample size, and needs more studies to test.

\section{Conclusion}

In the population scheduled for prostate biopsy for increased serum PSA or/and abnormal DRE with suspicious lesion on MRI but non-previous evidence of cancer, MRI/ TRUS fusion 3D-Tb combined with Sb significantly improves the PCa detection rate compared to each of them alone; MRI/TRUS fusion 3D-Tb detects a significantly more high-Gleason-score PCa, and tends to detect more $\mathrm{PCa}$ in the population with previous negative biopsy, but has no significant superiority in overall PCa detection.

\section{Limitation and Prospection}

In a subgroup analysis, only 3 cohorts enrolled men with negative biopsy analysis, resulting in some unclear outcomes. Therefore, more similar original studies with a large sample size were needed. Studies about men on active surveillance were excluded because we primarily focused on PCa screening. For men without suspicious lesion on MRI, systematic prostate biopsy could be guided by MRI/TRUS fusion 3D prostate model or traditional TRUS, but it needs further study. The MRI/TRUS could also be fused to $2 \mathrm{D}$ model automatically or visually, so the difference between 3D and 2D needs additional studies to test.

\section{Acknowledgment}

This study was funded by the Department of Science and Technology of Sichuan Province (CN; 2014JY0183).

\section{Disclosure Statement}

The authors report no direct or indirect commercial incentive associated with publishing this article.

\section{References}

1 Ouzzane A, Coloby P, Mignard JP, Allegre JP, Soulie M, Rebillard X, Salomon L, Villers A: [Recommendations for best practice for prostate biopsy]. Prog Urol 2011;21:18-28.

2 Takenaka A, Hara R, Ishimura T, Fujii T, Jo Y, Nagai A, Fujisawa M: A prospective randomized comparison of diagnostic efficacy between transperineal and transrectal 12-core prostate biopsy. Prostate Cancer Prostatic Dis 2008;11:134-138.

3 Carroll PR, Parsons JK, Andriole G, Bahnson RR, Castle EP, Catalona WJ, Dahl DM, Davis JW, Epstein JI, Etzioni RB, Farrington T, Hemstreet GP 3rd, Kawachi MH, Kim S, Lange PH, Loughlin KR, Lowrance W, Maroni P, Mohler J, Morgan TM, Moses KA, Nadler RB, Poch M, Scales C, Shaneyfelt TM, Smaldone MC, Sonn G, Sprenkle P, Vickers AJ, Wake R, Shead DA, Freedman-Cass DA: NCCN Guidelines Insights: Prostate Cancer Early Detection, Version 2.2016. J Nat Compr Canc Netw 2016;14: 509-519.

4 Raja J, Ramachandran N, Munneke G, Patel $\mathrm{U}$ : Current status of transrectal ultrasound- guided prostate biopsy in the diagnosis of prostate cancer. Clin Radiol 2006;61:142153.

5 Miano R, De Nunzio C, Kim FJ, Rocco B, Gontero P, Vicentini C, Micali S, Oderda M, Masciovecchio S, Asimakopoulos AD: Transperineal versus transrectal prostate biopsy for predicting the final laterality of prostate cancer: are they reliable enough to select patients for focal therapy? Results from a multicenter international study. Int Braz J Urol 2014;40: $16-22$.

6 Jones JS: Saturation biopsy for detecting and characterizing prostate cancer. BJU Int 2007; 99:1340-1344.

7 Lane BR, Zippe CD, Abouassaly R, Schoenfield L, Magi-Galluzzi C, Jones JS: Saturation technique does not decrease cancer detection during followup after initial prostate biopsy. J Urol 2008;179:1746-1750; discussion 1750.

8 Pereira RA, Costa RS, Muglia VF, Silva FF, Lajes JS, Dos Reis RB, Silva GE: Gleason score and tumor laterality in radical prostatectomy and transrectal ultrasound-guided biopsy of the prostate: a comparative study. Asian J Androl 2015; 17:815-820

9 Jo JK, Hong SK, Byun SS, Lee SE, Lee SE, Oh JJ: Prognostic significance of the disparity between biopsy and pathologic gleason score after radical prostatectomy in clinical candidates for active surveillance according to the royal marsden criteria. Clin Genitourin Cancer 2016; 14:e329-e333.

10 Zugor V, Poth S, Kühn R, Bernat MM, Porres D, Labanaris AP: Is an extended prostate biopsy scheme associated with an improvement in the accuracy between the biopsy gleason score and radical prostatectomy pathology? A multivariate analysis. Anticancer Res 2016; 36:4285-4288.

11 Siddiqui MM, Rais-Bahrami S, Truong H, Stamatakis L, Vourganti S, Nix J, Hoang AN, Walton-Diaz A, Shuch B, Weintraub M, Kruecker J, Amalou H, Turkbey B, Merino MJ, Choyke PL, Wood BJ, Pinto PA: Magnetic resonance imaging/ultrasound-fusion biopsy significantly upgrades prostate cancer versus systematic 12-core transrectal ultrasound biopsy. Eur Urol 2013;64:713-719. 
12 Shao W, Wu RY, Thng CH, Ling KV, Ng WS: Integrating MRI and MRSI information into trus-guided robotic prostate biopsy. Int J Humanoid Robotics 2006;3:499-522.

13 Kuru TH, Tulea C, Simpfendorfer T, Popeneciu V, Roethke M, Hadaschik BA, Hohenfellner M: MRI navigated stereotactic prostate biopsy. Fusion of MRI and real-time transrectal ultrasound images for perineal prostate biopsies. Urology 2012;51:50-56.

14 Cool DW, Romagnoli C, Izawa JI, Chin J, Gardi L, Tessier D, Mercado A, Mandel J, Ward AD, Fenster A: Comparison of prostate MRI-3D transrectal ultrasound fusion biopsy for first-time and repeat biopsy patients with previous atypical small acinar proliferation. Can Urol Assoc J 2016;10:342-348.

15 Delongchamps NB, Portalez D, Bruguiere E, Rouviere O, Malavaud B, Mozer P, Fiard G, Cornud F, Grp MS: Are magnetic resonance imaging-transrectal ultrasound guided targeted biopsies noninferior to transrectal ultrasound guided systematic biopsies for the detection of prostate cancer? J Urol 2016;196: 1069-1075.

16 Mendhiratta N, Rosenkrantz AB, Meng XS, Wysock JS, Fenstermaker M, Huang R, Deng FM, Melamed J, Zhou M, Huang WC, Lepor $\mathrm{H}$, Taneja SS: Magnetic resonance imagingultrasound fusion targeted prostate biopsy in a cnsecutive cohort of men with no previous biopsy: reduction of over detection through improved risk stratification. J Urol 2015;194: 1601-1606.

17 Siddiqui MM, Rais-Bahrami S, Turkbey B, George AK, Rothwax J, Shakir N, Okoro C, Raskolnikov D, Parnes HL, Linehan WM, Merino MJ, Simon RM, Choyke PL, Wood BJ, Pinto PA: Comparison of MRI/ultrasound fusion-guided biopsy with ultrasound-guided biopsy for the diagnosis of prostate cancer. J Am Med Assoc 2015;313:390-397.

18 Meng X, Rosenkrantz AB, Mendhiratta N, Fenstermaker M, Huang R, Wysock JS, Bjurlin MA, Marshall S, Deng FM, Zhou M, Melamed J, Huang WC, Lepor H, Taneja SS:
Relationship between prebiopsy multiparametric magnetic resonance imaging (MRI), biopsy indication, and MRI-ultrasound fusion-targeted prostate biopsy outcomes. Eur Urol 2016;69:512-517.

19 Ukimura O, Marien A, Palmer S, Villers A, Aron M, Abreu ALD, Leslie S, Shoji S, Matsugasumi T, Gross M, Dasgupta P, Gill IS: Trans-rectal ultrasound visibility of prostate lesions identified by magnetic resonance imaging increases accuracy of image-fusion targeted biopsies. World J Urol 2015;33:16691676.

20 Puech P, Rouviere O, Renard-Penna R, Villers A, Devos P, Colombel M, Bitker MO, Leroy X, Mege-Lechevallier F, Comperat E, Ouzzane A, Lemaitre L: Prostate cancer diagnosis: multiparametric MR-targeted biopsy with cognitive and transrectal US-MR fusion guidance versus systematic biopsy-prospective multicenter study. Radiology 2013;268:461-469.

21 Vourganti S, Rastinehad A, Yerram NK, Nix J, Volkin D, Hoang A, Turkbey B, Gupta GN, Kruecker J, Linehan WM, Choyke PL, Wood BJ, Pinto PA: Multiparametric magnetic resonance imaging and ultrasound fusion biopsy detect prostate cancer in patients with prior negative transrectal ultrasound biopsies. J Urol 2012;188:2152-2157.

22 Mendhiratta N, Meng XS, Rosenkrantz AB, Wysock JS, Fenstermaker M, Huang R, Deng FM, Melamed J, Zhou M, Huang WC, Lepor H, Taneja SS: Prebiopsy MRI and MRI-ultrasound fusion-targeted prostate biopsy in men with previous negative biopsies: impact on repeat biopsy strategies. Urology 2015;86:1192-1198.

23 Schoots IG, Roobol MJ, Nieboer D, Bangma $\mathrm{CH}$, Steyerberg EW, Hunink MGM: Magnetic resonance imaging-targeted biopsy may enhance the diagnostic accuracy of significant prostate cancer detection compared to standard transrectal ultrasound-guided biopsy: a systematic review and meta-analysis. Eur Urol 2015;68:438-450.

24 Klein J, de Gorski A, Benamran D, Vallee JP, De Perrot T, Wirth GJ, Iselin CE: Transrectal ultrasound-guided prostate biopsy for cancer detection: performance of 2D-, 3D- and 3DMRI fusion targeted techniques. Urol Int 2017;98:7-14.

25 Fiard G, Hohn N, Descotes JL, Rambeaud JJ, Troccaz J, Long JA: Targeted MRI-guided prostate biopsies for the detection of prostate cancer: initial clinical experience with realtime 3-dimensional transrectal ultrasound guidance and magnetic resonance/transrectal ultrasound image fusion. Urology 2013;81: 1372-1378.

26 NCCN: Clinical Practice Guidelines in Oncology. Prostate Cancer, Version 1. 2017. https://wwwnccnorg/professionals/physician_gls/f_guidelinesasp\#prostate, December 16. 2016.

27 Wein AJ, Kavoussi LR, Partin AW, Novick AC, Peters CA: Grade, Chaper 96: Pathology of Prostatic Neoplasia, Campbell-Walsh Uro$\log$, 10th Edition. Saunders, an imprint of Elsevier Inc., 2012:3831.

28 Kaplan I, Oldenburg NE, Meskell P, Blake M, Church P, Holupka EJ: Real time MRI-ultrasound image guided stereotactic prostate biopsy. Magn Reson Imaging 2002;20:295-299.

29 Kim EH, Vemana G, Johnson MH, Vetter JM, Rensing AJ, Strother MC, Fowler KJ, Andriole GL: Magnetic resonance imaging-targeted vs. conventional transrectal ultrasound-guided prostate biopsy: single-institution, matched cohort comparison. Urol Oncol 2015;33:109.e1-e6.

30 Oberlin DT, Casalino DD, Miller FH, Matulewicz RS, Perry KT, Nadler RB, Kundu S, Catalona WJ, Meeks JJ: Diagnostic value of guided biopsies: fusion and cognitive-registration magnetic resonance imaging versus conventional ultrasound biopsy of the prostate. Urology 2016;92:75-77.

31 Junker D, Schafer G, Heidegger I, Bektic J, Ladurner M, Jaschke W, Aigner F: Multiparametric magnetic resonance imaging/transrectal ultrasound fusion targeted biopsy of the prostate: preliminary results of a prospective single-centre study. Urol Int 2015;94:313-318. 